\title{
Photo-Rechargeable Organo-Halide Perovskite Batteries
}

Shahab Ahmad ${ }^{1, *}$, Chandramohan George ${ }^{1}$, David J. Beesley ${ }^{1}$, Jeremy J. Baumberg ${ }^{2}$ and Michael De Volder ${ }^{1} *$

${ }^{1}$ Institute for Manufacturing, Department of Engineering, University of Cambridge, Cambridge, CB3 OFS, United Kingdom.

${ }^{2}$ Nanophotonics Centre, Cavendish Laboratory, University of Cambridge, Cambridge, CB3 OHE, United Kingdom.

\begin{abstract}
:
Emerging autonomous electronic devices require increasingly compact energy generation and storage solutions. Merging these two functionalities in a single device would significantly increase their volumetric performance, however this is challenging due to material and manufacturing incompatibilities between solar energy harvesting and storage materials. Here we demonstrate that organic-inorganic hybrid perovskites can both generate and store energy in a rechargeable device termed a photo-battery. This photo-battery relies on highly photoactive two-dimensional lead halide perovskites to simultaneously achieve photo-charging and Li-ion storage. Integrating these functionalities provides simple autonomous power solutions while retaining capacities of up to $100 \mathrm{mAh} / \mathrm{g}$ and efficiencies similar to those of electrodes using a mixture of batteries and solar materials.
\end{abstract}

KEYWORDS: metal halide perovskite, layered-materials, Li-ion battery, photo-battery, selfrechargeable. 
The internet of things (IoT), smart cities, and other autonomous connected devices require continuous remote power sources for sensing, data processing, and communication ${ }^{1,2}$. To date, these power requirements are typically met by combining solar cells with rechargeable batteries. However, the use of two separate devices results in duplicated components and increased packaging requirements which adds to the device complexity, weight and $\operatorname{cost}^{3}$. More fundamentally, this leads to ohmic transport losses, and is sub-optimal because most solar cells have an open circuit voltage of 0.6 to $1.0 \mathrm{~V}$ which is insufficient to charge commercial Li-ion batteries, thus requiring additional DC-to-DC convertors or stacked cells ${ }^{4}$. Ongoing research efforts have improved the packaging efficiency by integrating batteries and solar cells in the same housing, as well as by developing designs where electrodes are shared between the battery and the solar cell ${ }^{5-8}$. These advances in device integration reduce the ohmic transport losses and increase the gravimetric energy density. However, co-assembling devices remains an imperfect manufacturing and scientific solution. Going some way to address these issues, photo-electrodes that consist of a physical mixture of solar energy harvesting and Li-ion storage materials have recently been reported ${ }^{9,10}$. Physically mixing photovoltaic and storage materials allows for compact device implementation, but suffers from other fundamental limitations: these include the mismatch of energy levels between the light-absorbing and storage materials resulting in inefficient charge transfer, the blocking of light by the storage medium so that less light reaches the light-absorbing material, reduced charge transport due to poor interfaces between the two materials, and manufacturing challenges such as the phase segregation of the two materials. It is therefore clear that a new material system is needed to address the above issues.

Paolella et al. have recently demonstrated light-assisted delithiation of lithium iron phosphate $\left(\mathrm{LiFePO}_{4}\right)$ nanocrystals in a two-electrode system where Li-metal is used as anode and a mixture of $\mathrm{LiFePO}_{4}$ and Ru dye (N719) as cathode ${ }^{9}$. In this work, the solar energy harvesting is performed by the dye, and energy storage by $\mathrm{LiFePO}_{4}$, resulting in photo-conversion and storage efficiencies of $0.06 \%$. In 2016, Sato et al. demonstrated a single anode material $\left(\mathrm{TiO}_{2}\right)$ integrated into a semi-transparent battery electrode ${ }^{33,34}$. While this is an elegant solution, $\mathrm{TiO}_{2}$ only absorbs light in the UV spectrum, and the cyclability, voltage window, and charge retention of these materials is rather limited. Miyasaka et al. reported a combined light conversion and energy storage device using soft carbonaceous materials where the photoactive layer is a thin LiI film is deposited on a mesoporous dye-filled $\mathrm{TiO}_{2}$ film ${ }^{7}$, but the output power of these three-electrode devices in 
sunlight is only $0.5 \mathrm{~mW}$ with an open circuit voltage $(\mathrm{OCV})$ of $0.45 \mathrm{~V}$. To the best of our knowledge, despite the above examples of photo-battery effects, the use of a single active material which can perform both photo-charging and energy storage processes efficiently has not been reported.

In recent years, 3D metal halide based perovskites have been studied intensively for use in solar cells because of their high conversion efficiencies of up to $22 \%{ }^{11-13}$. More recently, reports have suggested perovskites might also be used as anode materials in $\mathrm{Li}$-ion batteries ${ }^{21}$. However, these functionalities have never been combined in a single solar-battery device. Here we present the first report that polycrystalline metal-halide-based 2D perovskite materials, namely $\left(\mathrm{RNH}_{3}\right)_{2} \mathrm{MX}_{4}$ [R-organic, M-metal, X-halide], can combine both energy storage (battery functionality) and photo-charging (photovoltaic functionality) simultaneously (Figure 1a). This integration alleviates the above issues with composite devices and has the potential to open up an entirely new application domain for perovskites. 2D perovskites are used in this work because they self-assemble into very stable layered structures where inorganic monolayers of corner-sharing $\left[\mathrm{MX}_{6}\right]^{4-}$ octahedra are confined between interdigitating bilayers of organic cations (Figure $\left.1 \mathrm{~b}\right)^{20}$. Their enhanced stability compared to 3D systems is important for battery applications, but comes at the cost of a lower solar cell efficiency due to the inhibition of out-of-plane charge transport by the organic cations ${ }^{17-19}$. The optical absorption of the $\left(\mathrm{C}_{6} \mathrm{H}_{9} \mathrm{C}_{2} \mathrm{H}_{4} \mathrm{NH}_{3}\right)_{2} \mathrm{PbI}_{4}$ (hereafter CHPI) thin films show a characteristic strong exciton absorption peak at $508 \mathrm{~nm}$ with a broad band-to-band absorption around $380 \mathrm{~nm}$ (Figure 1c). Photoluminescence (PL) imaging of the perovskite films shows strong green emission, from the characteristic room temperature excitonic emission of CHPI (Figure 1d inset, excited with a $300 \mathrm{~nm}$ LED source, $\left.\lambda_{\mathrm{PL}} \sim 518 \mathrm{~nm}\right)^{20}$ 


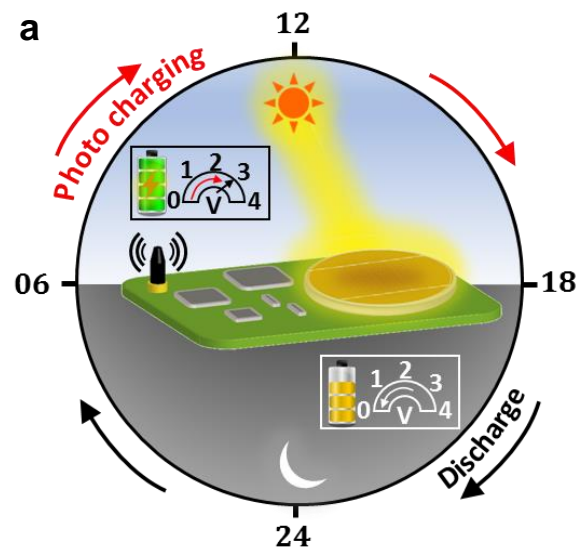

b

C

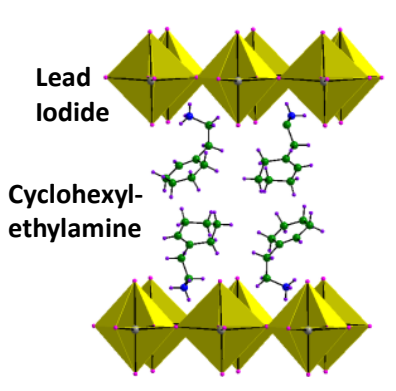

2D Perovskite

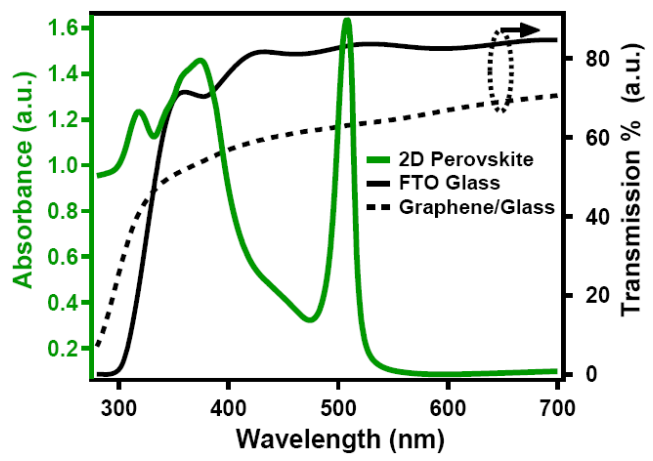

d

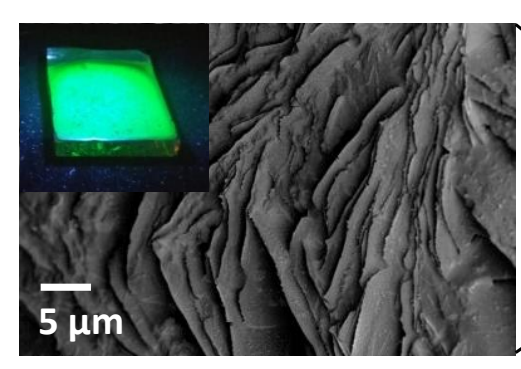

e

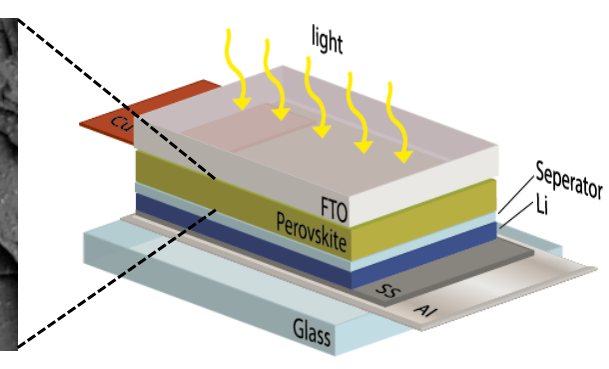

f

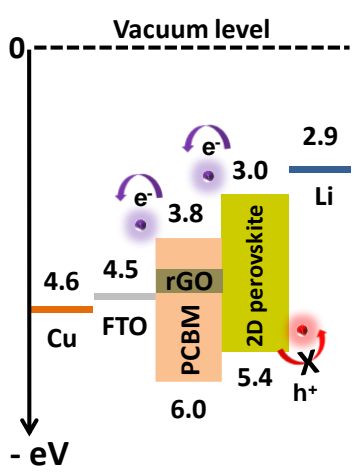

Figure 1. 2D Perovskite and fabrication of photo-battery. a, Schematic representation of the photo-battery concept. b, Crystal structure of 2D layered perovskites (CHPI). c, Optical absorption spectra of CHPI thin films and transmission of FTO glass and graphene substrates. $\boldsymbol{d}$, SEM image of drop-cast $2 D$ perovskite electrodes taken at $45^{\circ}$ tilt. The inset shows a PL image of the corresponding perovskite film ( $\lambda_{e x} \sim 300 \mathrm{~nm}$ LED source). $\boldsymbol{e}$, Schematic and $\boldsymbol{f}$, energy level diagram of perovskite photo-batteries.

The application of 2D perovskites for energy storage applications has not been reported previously. Therefore, we start by analyzing the performance of 2D perovskites as a battery material in standard coin cell configurations (see Methods). The morphology of CHPI at the microscale is key for both the battery and solar performance, therefore, instead of using standard spin coating, which results in horizontal stacking of the 2D perovskite platelets (see Supporting Information, Figure $\mathrm{S} 1 \mathrm{a}, \mathrm{b})^{20}$, we drop-cast the perovskite solution blended with conductive additives (reduced graphene oxide, rGO) and a binder (PVDF) which results in vertically-aligned nanoplatelets ( 320 nm thick and $\sim 8-10 \mu \mathrm{m}$ high, Figure 1d and Supporting Information, Figure S1c). Since these vertically aligned perovskite nanoplatelets are grown directly on the surface of the FTO 
substrate, there is a good interface and charge transfer from the perovskite film to the FTO. Thick films with vertically-aligned crystals introduce porosity, and improve light interaction when compared to pristine CHPI thin films (transmittance $50 \%$ versus 14\%, see Supporting Information, Figure S1d). At the same time, rGO improves the electrical transport within the film and PVDF enhances the mechanical stability of the cell. Other carbon-based conductive additives such as carbon nanotubes (CNTs) also produce functioning cells but of poorer cyclability and lower efficiency. A likely reason for the better performance of rGO is the lower work function of rGO ($4.4 \mathrm{eV})$ compared to CNTs $(-4.8 \mathrm{eV})$ which reduces the energy barrier for photo-electron transport from perovskite to rGO (Figure 1f). XRD measurements (see Supporting Information, Figure $\mathrm{S} 1 \mathrm{e}, \mathrm{f})$ show that the presence of rGO in the perovskite film does not affect the intralayer $d$-spacing, which suggests the rGO is located in-between the perovskite crystals. Charge-discharge potential curves of coin cell (non-photo-chargeable) batteries (see Supporting Information, Figure S2a,b) show that lead-iodide perovskites offer capacities of $\sim 90-100 \mathrm{mAh} / \mathrm{g}$ in the first cycle and 2D lead bromide $\left(\left(\mathrm{C}_{6} \mathrm{H}_{9} \mathrm{C}_{2} \mathrm{H}_{4} \mathrm{NH}_{3}\right)_{2} \mathrm{PbBr}_{4}\right.$, hereafter $\left.\mathrm{CHPB}\right)$ perovskite cells achieve up to $410 \mathrm{mAh} / \mathrm{g}$, which is higher than the graphite anodes $(\sim 370 \mathrm{mAh} / \mathrm{g})$ used in commercial Li-ion batteries. In spite of exhibiting useful capacity these perovskite materials presently suffer from stability issues such that their capacity drops to a fifth of its initial value within a few galvanostatic chargedischarge cycles (see Supporting Information, Figure S2b). This accords with recent reports on non-photo-rechargeable 3D perovskites batteries ${ }^{21}$. Indeed these stability issues are being addressed intensely by the perovskite community for various other applications ${ }^{22}$.

A hybrid device architecture is designed to achieve the above-mentioned simultaneous solar-battery functionality (Figure 1e). Briefly, a 2D perovskite-rGO-PVDF film is sandwiched between a separator (frit) and a transparent collector electrode (here fluorine-doped tin oxide, FTO, see Methods). For energetically favorable transport of electrons from the perovskite to the electron-selective electrode (Cu-foil), either rGO or PCBM are used as electron transport materials (see Methods and Figure 1f). A mesh-like Cu electrode could be used as an alternative for FTO, but this would block part of light and reduce the overall performance of the device therefore in our photo-battery devices we have used FTO connected with $\mathrm{Cu}$-metal foil, as shown in Figure 1e. Supporting Information, Figure S4). Photo-batteries are first charged to saturation by a broadband light source $\left(\lambda \sim 420-650 \mathrm{~nm}\right.$, intensity $100 \mathrm{~mW} / \mathrm{cm}^{2}$, see Supporting Information, Figure S3) and then discharged through an electrical resistor of $21.5 \mathrm{k} \Omega$ (or an LED if mentioned). Initially, the 
photo-battery with PCBM as conducting additive (structure B1, see Methods), is photocharged up to $3.05 \mathrm{~V}$ and then discharged to $0.4 \mathrm{~V}$. However, we found that below $1.4 \mathrm{~V}$, the typical dark yellow color of the CHPI electrodes turns black (see Supporting Information, Figure S4a,b). To confirm that the change in colour is not due to lithiation of the FTO, it is replaced by graphene, but the colour change persists (see Supporting Information, Figure S5). Further, at low discharge voltages, post-mortem Fourier transform infrared spectroscopy (FTIR) suggests excessive electrolyte decomposition or solid electrolyte interphase (SEI) formation (see Supporting Information, Figure S6) $24,23,31$. Therefore, we limit the discharge voltage to $1.4 \mathrm{~V}$ in what follows.
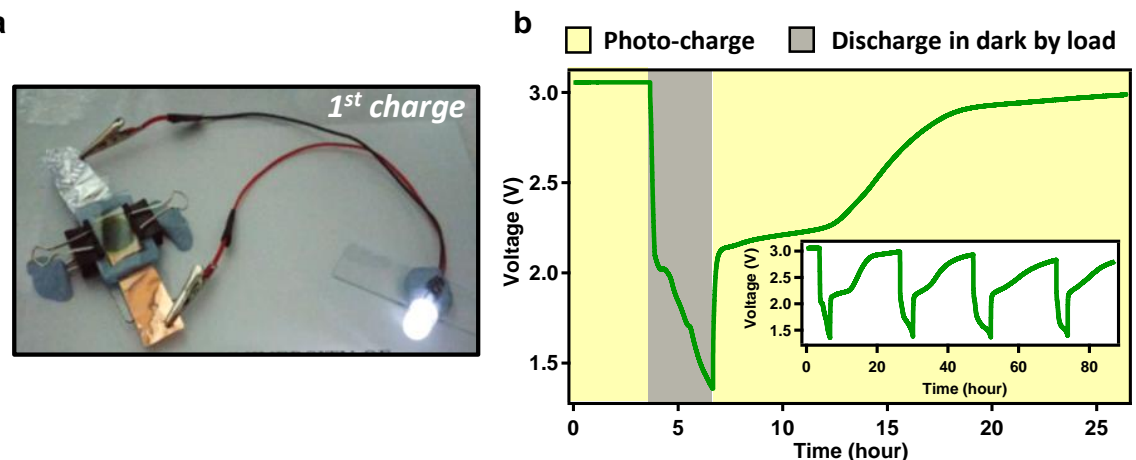

C

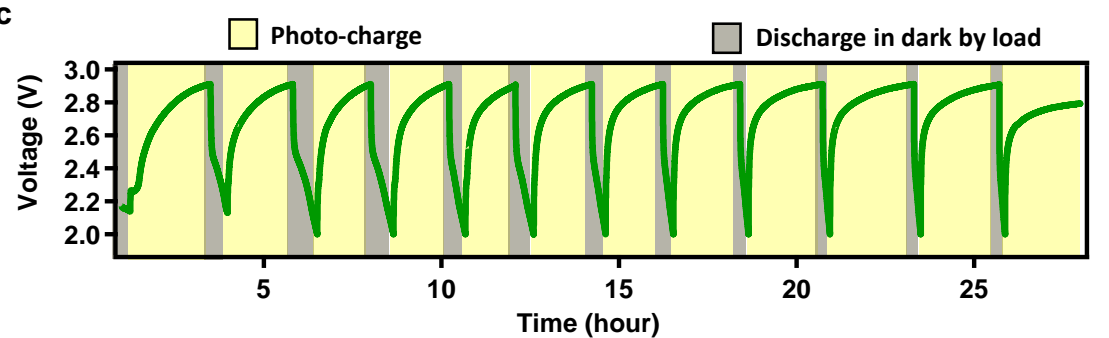

d

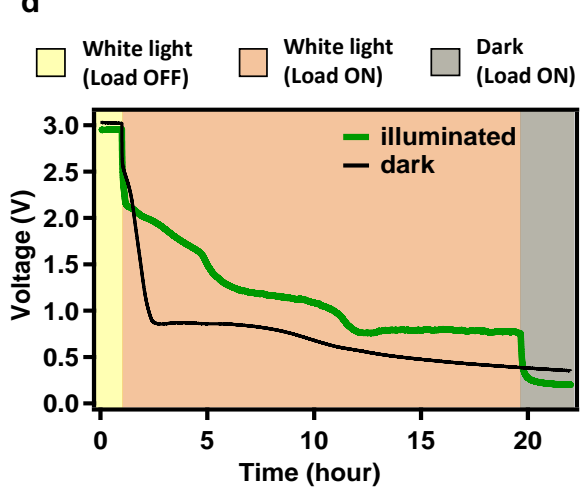

e
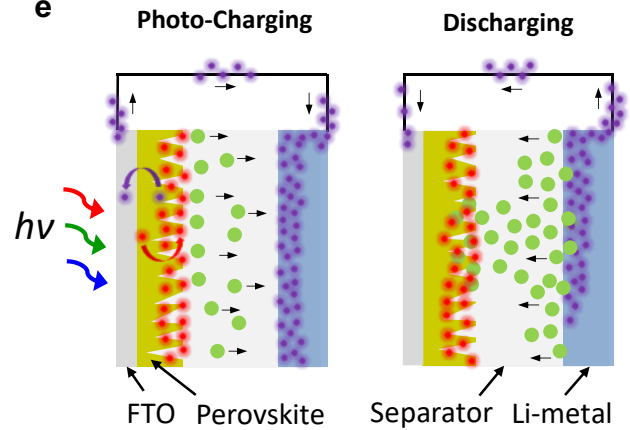

- electron $\bullet$ hole Li-ion

Figure 2. Perovskite photo-battery performance and mechanism. a, Photograph of a $3 V$ LED powered by a CHPI photo-battery after the $1^{\text {st }}$ cycle of photo-charging. $\boldsymbol{b}$, First photo-charge (broadband light $100 \mathrm{~mW} / \mathrm{cm}^{2}$ ) and discharge (dark, $21.5 \mathrm{k} \Omega$ load) voltage profile of a CHPIbased photo-battery. The inset shows further cycling of the photo-battery under similar conditions. 
$\boldsymbol{c}$, Photo-battery cycling over a limited potential range. $\boldsymbol{d}$, Potential discharge curve for photobatteries in dark (black) and illuminated (green) conditions. $\boldsymbol{e}$, Schematics of the photo-charge generation, transfer, and storage mechanisms in perovskite photo-batteries.

Figure 2 shows the photo-battery performance in more detail. First, we find that our fully lightcharged devices can power a commercial $3 \mathrm{~V}$ white light LED (operating at electrical power of $\sim 0.6 \mathrm{~mW}$ ) for more than an hour (photograph Figure 2a and Supporting Information, Figure S7a). More energy can be stored by simply using larger area electrodes since the photo-battery perovskite electrode is insensitive to defects from scaling. Further, these photo-batteries can run over the span of a full week, which is encouraging in comparison to the lifetime of other perovskite systems $^{25}$. Since the photo-batteries are charged with light and discharged over a resistor, the charge and discharge cycles are very different, and we estimate the Coulombic efficiency (CE) to be $17 \%$ in the first galvanostatic charge-discharge tests in the full potential window. Further, we find a drop in voltage of less than $10 \%$ over a span of 13 hours for photo-charged batteries left in dark conditions (see Supporting Information, Figure S7b). When testing the devices between 3.0 and $1.4 \mathrm{~V}$, good cyclability is achieved, with only a slight decay in the photo-charging potential (Figure 2b, with rGO conducting additive, see B2 in Methods). When cycled between 2.95 and 2.0 $\mathrm{V}$, no decay in the photo-charging potential is observed after more than 10 cycles. However, the capacity of the electrode shows some fading (Figure 2c), as expected based on the initial coin-cell results (see Supporting Information, Figure S2c). In what follows, we will investigate the storage and capacity fading mechanism in more detail. Next, two batteries (structure B2) are discharged through identical loads $(21.5 \mathrm{k} \Omega)$ with the first battery in dark conditions and the second one illuminated $\left(100 \mathrm{~mW} / \mathrm{cm}^{2}\right)$. As shown in Figure 2d, the latter maintains an overall higher output potential along with an almost two times longer discharge time from 3.0 to $1.4 \mathrm{~V}$. Further, a plateau at $\sim 1.1 \mathrm{~V}$ is observed before stabilizing at $0.8 \mathrm{~V}$. At this stage, the battery functionality is depleted, and the device relies only on photo-generated charge carriers (conversion efficiency, $\eta \sim 0.034 \%$ ). This is confirmed by the sudden drop to $0.2 \mathrm{~V}$ when the light is turned off after 19 hours (decay time $\sim 14$ mins, grey region in Figure 2d). This data further confirms the ability of CHPI to simultaneously operate as a battery and a photo harvesting material. Alternative photocharge/discharge curves in light and dark conditions are reported in Supporting Information, Figure S8. 

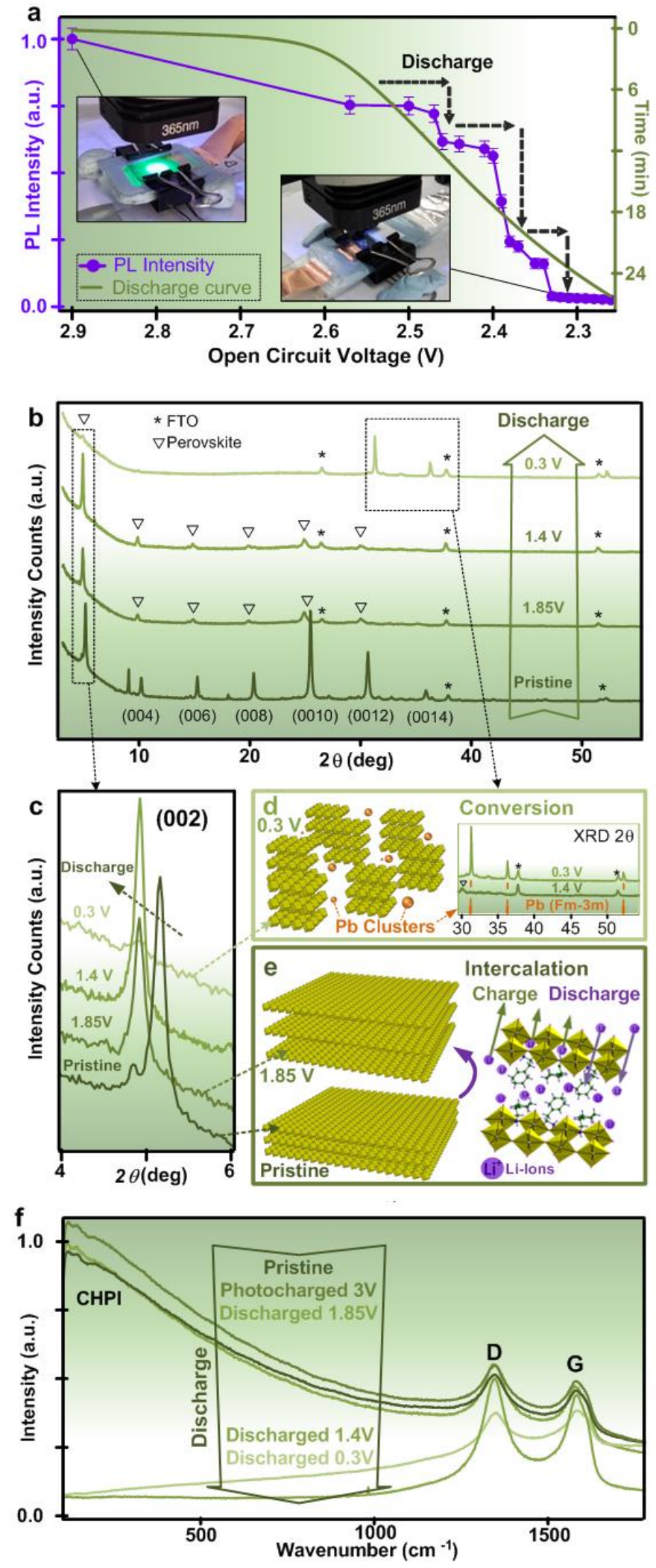

Figure 3. In-situ measurements. a, In-situ confocal PL versus measured open circuit voltage while discharging through a resistive load (dashed arrows added to guide the eye). Insets show 
the PL response ( $\lambda_{e x} \sim 365 \mathrm{~nm} L E D$ source) of the photo-battery when photo-charged to $2.90 \mathrm{~V}$ (left) and after discharge to $2.26 \mathrm{~V}$ (right). $\boldsymbol{b}, X R D$ patterns of photo-battery electrodes (B2) discharged at different potential levels, with XRD of pristine CHPI thin film also added for comparison (shifted along y-axis for clarity). c, Close-up view of characteristic peak (002) of CHPI, showing a shift towards lower diffraction angle upon lithiation. $d$, Schematic representation of Pb-metal cluster formation in case of perovskite photo-battery electrode discharged to deep potential levels. Inset XRD spectra of electrode discharged to $0.3 \mathrm{~V}$ matches well with lead metal XRD peaks (space group: Fm-3m shown in orange). e, Layered crystal structure of CHPI illustrating increase in $d$ spacing upon Li-ion intercalation between PbI $\mathrm{I}_{6}$ octahedra. $f$, Micro-Raman spectra of CHPI electrodes at different discharge levels.

In what follows, we elucidate both the energy storage and the photo-charging mechanisms of these devices. As suggested in a recent report of DFT simulations of 3D organic-inorganic hybrid perovskite batteries, we believe that energy is stored in our $2 \mathrm{D}$ perovskites by a combination of an intercalation and conversion mechanism ${ }^{32}$. We first study the storage mechanism at low discharge levels using in-situ confocal PL measurements $\left(\lambda_{\mathrm{PL}} \sim 518 \mathrm{~nm}\right.$, diode laser excitation source, $\lambda_{\mathrm{ex}} \sim 447 \mathrm{~nm}$, see Supporting Information, Figure S9). Figure 3a shows that the bright green PL of the photo-charged devices is quenched when discharging to $2.26 \mathrm{~V}$, and the exciton PL intensity decreases non-linearly similar to the potential discharge curve (Figure 2d). These in-situ measurements suggest that the discharge process modifies the perovskite structure, which we investigate further by post-mortem X-ray diffraction (XRD) (Figure 3b). These measurements show a shift in the CHPI characteristic (002) plane peak $\left(2 \boldsymbol{\theta} \sim 5.2^{\circ}\right)$ towards lower diffraction angles for electrodes discharged to $1.85 \mathrm{~V}$ or less $\left(2 \boldsymbol{\theta} \sim 4.9^{\circ}\right)$. This implies that the $d$-spacing between the metal halide atomic interlayers increases from $17 \AA$, for pristine electrodes, to $18 \AA$ when discharged to $1.85 \mathrm{~V}$ or lower. This expansion in the $d$-spacing suggests that Li-ions are inserted between the metal halide atomic interlayers ${ }^{32}$. The change in $d$-spacing during lithiation also suggest that photo-charging is not due to surface reactions but it is a bulk process taking place in the bulk of the perovskite. This Li-ion migration in CHPI can follow different paths (ionic radius $\sim 0.76 \AA$ ), for instance through the spaces between lead halide octahedrons (>37 $\AA^{2}$ ) along the $c$ axis of the triclinic unit cell of 2D-perovskite $\operatorname{crystal}^{28}$ (see Figure 3c). This is similar to organic 
moieties which travel between adjacent $\mathrm{PbI}_{6}$ octahedrons to form the $2 \mathrm{D}$ perovskites crystals ${ }^{29}$ during initial intercalation of the organics into $\mathrm{PbI}_{2}$.

At voltages below $1.4 \mathrm{~V}$, both the perovskite peaks disappeared both in the XRD patterns (Figure 3b) and Raman spectra (Figure 3f), indicating a more substantial change in the material structure. Further, at $0.3 \mathrm{~V}$ new XRD peaks appear, which match well with $\mathrm{Pb}$ (space group Fm$3 m$, Figure $3 \mathrm{~d}$ ) and is likely the result of a conversion reaction similar to one suggested recently for 3D perovskites (Eqn 1) $)^{32}$ :

$$
2 \mathrm{Li}+\left(\mathrm{C}_{6} \mathrm{H}_{9} \mathrm{C}_{2} \mathrm{H}_{4} \mathrm{NH}_{3}\right)_{2} \mathrm{PbI}_{4} \rightarrow 2\left(\mathrm{C}_{6} \mathrm{H}_{9} \mathrm{C}_{2} \mathrm{H}_{4} \mathrm{NH}_{3} \mathrm{I}\right)+2 \mathrm{LiI}+\mathrm{Pb}(m)
$$

During the photo charging process, the alternating 2D semiconducting inorganic and insulating organic monolayers act as potential wells and barriers respectively, resulting in electronic and dielectric confinement effects ${ }^{14-16}$, and generation of excitons upon illumination with photons exceeding the exciton energy $\left(\lambda_{x} \sim 508 \mathrm{~nm}\right)$. The photo-generated electrons are readily collected by the FTO electrode due to the energetically favorable landscape depicted in Figure $1 \mathrm{f}$ (perovskite/rGO (or PCBM)/FTO/Cu) ${ }^{15,26}$. These photo-electrons accumulate on the counterelectrode through the external circuit (Figure 2e, photo-charging), and we hypothesize that in the intercalation regime (low discharge), the photo-generated holes provide sufficient repulsion to drive Li-ions out of the perovskite matrix. This could then induce the photo-charging of the battery as sketched in Figure 2e. The blend of perovskite with rGO/PCBM provides extensive conducting pathways through grain boundaries and interfaces, however short carrier lifetimes ( 200 ps) and short diffusion lengths $(<100 \mathrm{~nm})$ of electrons and holes in 2D perovskites mean most either recombine or are trapped ${ }^{27}$. At lower discharges in the conversion region, we also observe some recharging (see Supporting Information, Figure S4), which may be due some remaining intercalation effects, or due to the photo-generated holes reacting with the lithium iodide (LiI) to form $\mathrm{Li}^{+}$:

$$
2 \mathrm{LiI}+2 h^{+} \rightarrow 2 \mathrm{Li}^{+}+\mathrm{I}_{2}
$$

The $\mathrm{Li}^{+}$is further reduced at the counter electrode by the accumulated photo-electrons $\left(\mathrm{Li}^{+}+e^{-} \rightarrow\right.$ Li), thus balancing the total generated photo-charge carriers and inducing photo-charging of the battery as sketched in Figure 2e. The capacity fading observed in the charge-discharge curves of the photo-batteries (see Figure $2 \mathrm{~b}$ and $\mathrm{c}$ ), is probably due to a number of different factors including possibly deterioration of the material morphology during charging and discharging, the formation of insulating solid-electrolyte-interfaces (SEI) and the formation of poorly-reversible lead metal 
$\mathrm{Pb}(m)$ (see chemical reaction 1 ). The photo-inactive $\mathrm{Pb}(m)$ can alloy with $\mathrm{Li}$ to form $\mathrm{Li}_{\mathrm{x}} \mathrm{Pb}$ $(0<x<4.4)$ resulting in large volume increase, and likely cracking and crumbling of the material ${ }^{35}$. This results in the loss of electrical contacts and hinders the reversibility of the reaction with $\mathrm{Li}$, hence causes further capacity fading. Nevertheless, our system is relatively stable compared to other solar-chargeable energy storage devices ${ }^{7,9,33,34}$, and further improvements are possible by replacing $\mathrm{Pb}$ in the perovskite structure with other metals, such as $\mathrm{Sn}, \mathrm{Bi}$ to provide better battery cycling. We note changes in elemental composition of the perovskite (in both $2 \mathrm{D}$ and 3D perovskites) cause changes in the optical band gap, which will change the performance of the photo-battery. Perovskite photo-batteries with alternative compositions should follow similar energy storage mechanisms this may enable further device optimization. Overall, the above analysis underpins the unique capability of CHPI to combine both energy storage and photocharging mechanisms in the same material. This may lead to entirely new application domains for perovskites.

To date, batteries rely on external power sources to charge them. Our work demonstrates that highly photoactive 2D lead halide perovskites can simultaneously store energy and recharge by light. This paves the way for more compact integrated off-grid energy sources, which could profit in the longer term from fewer internal losses and lower weight than present separate solar cell/battery solutions. Our photo-battery devices already show efficiencies comparable to those of electrodes using mixtures of solar cells and batteries and storage capacities of $100 \mathrm{mAh} / \mathrm{g}$. Further, these materials can be solution processed which leads to straight-forward scale-up strategies once more research is conducted to enhance their efficiency and stability.

\section{EXPERIMENTAL SECTION}

\section{Methods}

Materials. The following chemicals were purchased from Sigma-Aldrich: Lead iodide ( $\left.\mathrm{PbI}_{2}\right)$, lead bromide $\left(\mathrm{PbBr}_{2}\right)$, hydroiodic acid $(\mathrm{HI})$, hydrobromic acid ( $\left.\mathrm{HBr}\right)$, cyclo-hexyl-ethylamine $\left(\mathrm{C}_{6} \mathrm{H}_{9} \mathrm{C}_{2} \mathrm{H}_{4} \mathrm{NH}_{2}\right)$, reduced graphene oxide ( $\left.\mathrm{rGO}\right)$, electrochemical grade propylene carbonate, ethylene carbonate, diethyl carbonate, N-methyl-2-pyrrolidone, polyvinylidene fluoride (PVDF) and metal foils ( $\mathrm{Al}, \mathrm{Cu}$ and $\mathrm{Li}$ ). Polypropylene layers were purchased from Cell Guard. The 
phenyl-C61 butyric acid methyl ester (PCBM) of $>99.5 \%$ purity grade was purchased from Solenne B.V.

Synthesis of perovskites. The synthesis of 2D perovskite is performed by using a sol-gel method reported previously ${ }^{19,30}$. First $\left(\mathrm{C}_{6} \mathrm{H}_{9} \mathrm{C}_{2} \mathrm{H}_{4} \mathrm{NH}_{3}\right) \mathrm{X}(\mathrm{X}=\mathrm{I}$ or $\mathrm{Br})$ was synthesized from the mixture of 1:1 molar ratio of aqueous solutions of $\mathrm{HX}$ ( $\mathrm{HI}$ or $\mathrm{HBr}$ ) and cyclo-hexyl-ethylamine $\left(\mathrm{C}_{6} \mathrm{H}_{9} \mathrm{C}_{2} \mathrm{H}_{4} \mathrm{NH}_{2}\right)\left(100^{\circ} \mathrm{C}\right.$ and stirring at $\left.2000 \mathrm{rpm}\right)$. The precipitate from the reactant solution is separated and then washed with diethyl ether. To synthesize 2D perovskites $\left(\left(\mathrm{C}_{6} \mathrm{H}_{9} \mathrm{C}_{2} \mathrm{H}_{4} \mathrm{NH}_{3}\right)_{2} \mathrm{PbX}_{4}\right)$, stoichiometric amounts of $\left(\mathrm{C}_{6} \mathrm{H}_{9} \mathrm{C}_{2} \mathrm{H}_{4} \mathrm{NH}_{3}\right) \mathrm{X}$ and corresponding lead halide $\left(\mathrm{PbI}_{2}\right.$ or $\left.\mathrm{PbBr}_{2}\right)$ were dissolved in $\mathrm{N}, \mathrm{N}$-dimethylformamide (DMF) (hereafter, the resultant compounds are referred to as CHPI and CHPB, respectively). Perovskite powder is extracted from the above prepared solution by drying overnight in a vacuum oven at $60^{\circ} \mathrm{C}$.

Preparation of electrodes. Perovskite photo-battery electrodes were prepared by using different additives, rGO and PCBM. For both additives, lead halide perovskites are made following a similar recipe. $10 \mathrm{mg}$ of rGO or PCBM is dissolved in $1 \mathrm{ml} \mathrm{N}$-methy-2-pyrrolidone (NMP, in glove box) and sonicated for 1 hour. $85 \mathrm{mg}$ of perovskite is dissolved in the same solution and stirred overnight. Then, $5 \mathrm{mg}$ of polyvinylidene fluoride (PVDF) binder is added followed by 2 hours of stirring. CHPI electrodes using PCBM/PVDF as additives are referred to as $B 1$ and $\mathrm{rGO} / \mathrm{PVDF}$ as $B 2$ respectively. Both $B 1$ and $B 2$ were transferred in a glove box to prepare the electrodes. $60 \mu 1$ of $B 1$ or $B 2$ is drop-casted on pre-cleaned and UV-ozone treated FTO substrates and left on a hotplate for drying overnight at $45^{\circ} \mathrm{C}$. All devices are made on FTO substrates with an area of $1.0 \mathrm{~cm}$ $\mathrm{x} 1.5 \mathrm{~cm}$.

Fabrication of the photo-batteries. Perovskite photo-batteries are assembled in an Ar filled glovebox. Al-metal foil and Li-metal foil $(25 \mu \mathrm{m})$ are stacked on stainless steel (SS) disks. Whatman borosilicate paper, soaked with $1 \mathrm{M} \mathrm{LiPF}_{6}$ as electrolyte, is placed on Li-metal as a separator. On top of the separator, the perovskite electrodes with a $\mathrm{Cu}$-foil extended electrode are gently flipped and covered with another glass slide $(2.5 \mathrm{~cm}$ x $3.7 \mathrm{~cm})$ to hold all layers together. Finally, the edges were sealed and clips were used to maintain the interfacial contacts between all components of device. 
Characterizations. All photo-battery measurements were performed in air by using a Biologic VMP-3 galvanostat. For photo-charging, an LED-based broadband $(\lambda \sim 420-650 \mathrm{~nm}$, intensity $100 \mathrm{~mW} / \mathrm{cm}^{2}$ ) source is used for irradiation. Open circuit voltage (OCV) is measured to obtain the photo-charge and discharge potential curves. For discharge, the photo-battery is connected to either a resistor of $21.5 \mathrm{k} \Omega$ load or a commercial $3 \mathrm{~V}$ white light LED. A $447 \mathrm{~nm}$ diode laser is coupled to an Olympus BX-51 microscope for confocal PL measurements. For PL imaging, deep UV (300 nm, Thorlabs-M300L4) and UV (365nm, Thorlabs-M365L2) mounted LEDs are used. For post-mortem analysis, the photo-batteries are opened in an Ar-glove box and dried overnight at $40{ }^{\circ} \mathrm{C}$. The XRD measurement are performed on a Bruker D8, Raman on a Renishaw InVia Raman Microscope, and FTIR measurements on a Perkin Elmer Frontier. 


\section{ASSOCIATED CONTENT}

\section{Supporting information}

The Supporting Information is available free of charge on the ACS Publications website at DOI:

\section{AUTHOR INFORMATION}

\section{Correspondence Authors}

*E-mail: $\underline{\text { sa754@cam.ac.uk }}$

*E-mail: $\underline{\text { mfld2@cam.ac.uk }}$

\section{Present Address}

S.A.: Centre for Nanoscience and Nanotechnology, Jamia Millia Islamia (A Central University), New Delhi, India 110025. E-mail: sahmad28@jmi.ac.in

\section{Notes}

The authors declare no competing financial interest.

\section{ACKNOWLEDGEMENTS}

Authors acknowledge support from Dr. A. Sadhanala, Optoelectronics Group, and Marie-Elena Kleemann, Nanophotonics Centre, for assistance with measurements. This work was funded by an ERC Starting Grant (HIENA -337739), ERC (LINASS, 320503), and EPSRC (EP/L027151/1).

\section{REFERENCES}

1. Haight, R., Haensch, W. \& Friedman, D. Solar-powering the Internet of Things. Science353,124-125 (2016).

2. Ahmad, S., Copic, D., George, C. \& De Volder, M. Hierarchical Assemblies of Carbon Nanotubes for Ultraflexible Li-Ion Batteries. Adv. Mater.28, 6705-6710 (2016).

3. Vlad, A., Singh, N., Galande, C. \& Ajayan, P. M. Design Considerations for Unconventional Electrochemical Energy Storage Architectures. Adv. Energy Mater.5, 1402115 (2015).

4. $\mathrm{Xu}$, J. et al. Efficiently photo-charging lithium-ion battery by perovskite solar cell. Nat. Commun.6, 8103 (2015). 
5. $\mathrm{Xu}$, J. et al. Integrated Photo-supercapacitor Based on Bi-polar $\mathrm{TiO}_{2} \mathrm{Nanotube} \mathrm{Arrays}$ with Selective One-Side Plasma-Assisted Hydrogenation. Adv. Funct. Mater.24, 18401846 (2014).

6. Méndez, M. A., Peljo, P., Scanlon, M. D., Vrubel, H. \& Girault, H. H. Photo-Ionic Cells: Two Solutions to Store Solar Energy and Generate Electricity on Demand. J. Phys. Chem. C118, 16872-16883 (2014).

7. Miyasaka, T., Ikeda, N., Murakami, T. N. \& Teshima, K. Light Energy Conversion and Storage with Soft Carbonaceous Materials that Solidify Mesoscopic Electrochemical Interfaces. Chem. Lett.36, 480-487 (2007).

8. Thimmappa, R. et al. A Chemically Chargeable Photo Battery. J. Phys. Chem. C119,14010-14016 (2015).

9. Paolella, A. et al.Light-assisted delithiation of lithium iron phosphate nanocrystals towards photo-rechargeable Li-ion batteries. Nat. Comm. 8, 14643 (2017).

10. Nguyen, O. et al. Shedding light on the light-driven lithium ion de- insertion reaction: towards the design of a photo- rechargeable battery. J. Mat. Chem. A (2017). doi:10.1039/c7ta00493a

11. Saliba, M. et al. Incorporation of rubidium cations into perovskite solar cells improves photovoltaic performance. Science (2016) (aah5557).

12. Akkerman, Q. A. et al. Strongly emissive perovskite nanocrystal inks for high-voltage solar cells. Nat. Energy2, 16194 (2016).

13. Stranks, S. D. \& Snaith, H. J. Metal-halide perovskites for photovoltaic and light-emitting devices. Nat. Nanotechnol.10, 391-402 (2015).

14. Ishihara, T., Takahashi, J. \& Goto, T. Optical properties due to electronic transitions in two-dimensional semiconductors $\left(\mathrm{C}_{\mathrm{n}} \mathrm{H}_{2 \mathrm{n}+1} \mathrm{NH}_{3}\right)_{2} \mathrm{PbI}_{4}$. Phys. Rev. B42,11099-11107 (1990).

15. Ishihara, T. Optical properties of PbI-based perovskite structures. J. Lumin.60-61, 269274 (1994).

16. Dou, L. et al. Atomically thin two-dimensional organic-inorganic hybrid perovskites. Science349, 1518-1521 (2015).

17. Ahmad, S. et al. Strong photocurrent from 2D excitons in solution-processed stacked perovskite semiconductor sheets. ACS Appl. Mater. Interfaces7, 25227-25236(2015). 
18. Cao, D. H., Stoumpos, C. C., Farha, O. K., Hupp, J. T. \& Kanatzidis, M. G. 2D Homologous Perovskites as Light-Absorbing Materials for Solar Cell Applications. J. Am. Chem. Soc.137, 7843-7850 (2015).

19. Safdari, M. et al. Layered 2D alkyldiammonium lead iodide perovskites: synthesis, characterization, and use in solar cells. J. Mater. Chem. A4, 15638-15646 (2016).

20. Ahmad, S. \& Prakash, G. V. Strong room-temperature ultraviolet to red excitons from inorganic organic-layered perovskites, $\left(\mathrm{R}-\mathrm{NH}_{3}\right)_{2} \mathrm{MX}_{4}\left(\mathrm{M}=\mathrm{Pb}^{2+}, \mathrm{Sn}^{2+}, \mathrm{Hg}^{2+} ; \mathrm{X}=\mathrm{I}^{-}, \mathrm{Br}^{-}\right) . J$. Nanophotonics 8,083892 (2014).

21. Xia, H.-R. et al. Hydrothermal synthesis of organometal halide perovskites for Li-ion batteries. Chem. Commun.51, 13787-13790 (2015).

22. Tai, Q. et al. Efficient and stable perovskite solar cells prepared in ambient air irrespective of the humidity. Nat. Commun.7, 11105 (2016).

23. Jiang, Q. et al. Electrochemical Doping of Halide Perovskites with Ion Intercalation. ACS Nano11,1073-1079 (2017).

24. Cabana, J., Monconduit, L., Larcher, D. \& Palacín, M. R. Beyond Intercalation-Based LiIon Batteries: The State of the Art and Challenges of Electrode Materials Reacting Through Conversion Reactions. Adv. Mater.22, E170-E192 (2010).

25. Zhao, X. \& Park, N.-G. Stability Issues on Perovskite Solar Cells. Photonics2, 1139-1151 (2015).

26. Tsai, H. et al. High-efficiency two-dimensional Ruddlesden-Popper perovskite solar cells. Nature536, 312-316 (2016).

27. Milot, R. L. et al. Charge-Carrier Dynamics in 2D Hybrid Metal-Halide Perovskites. Nano Lett.16, 7001-7007 (2016).

28. Billing, D. G., Lemmerer, A. Poly[bis[2-(1-cyclohexenyl)ethylammonium] di- $\mu$-iododiodoplumbate(II)]. Acta Crystallogr. Sect. C Cryst. Struct. Commun.62, m269-m271 (2006).

29. Ahmad, S., Kanaujia, P. K., Niu, W., Baumberg, J. J. \& Vijaya Prakash, G. In situ intercalation dynamics in inorganic-organic layered perovskite thin films. ACS Appl. Mater. Interfaces 6, 10238-10247 (2014).

30. Mitzi, D. B. in Synthesis, Structure, and Properties of Organic-Inorganic Perovskites and Related Materials. John Wiley \& Sons, Inc., pp. 1-121 (John Wiley \& Sons, Inc.). 
31. Zhuang, G. V. et al., A Study of Electrochemical Reduction of Ethylene and Propylene Carbonate Electrolytes on Graphite Using ATR-FTIR Spectroscopy. Electrochemical and Solid-State Letters, 8, A441-A445 (2005).

32. Dawson, J. A. et al., Mechanisms of Lithium Intercalation and Conversion Processes in Organic-inorganic Halide Perovskites. ACS Energy Lett. 1-8 (2017).

33. Nagai, H., \& Sato, M. Highly Functionalized Lithium-Ion Battery, Alkali-ion Batteries, Dr. Dongfang Yang (Ed.). InTech, Chapter 6 (2016). DOI: 10.5772/63491.

34. Nagai, H., Suzuki, T., Takahashi, Y. \& Sato, M. Photovoltaic lithium ion battery fabricated by molecular precursor method. Funct. Mater. Lett. 9, 1650046 (2016).

35. Martos, J., Morales, J., Sanchez, L. Lead-based systems as suitable anode materials for Liion batteries. Electrochimica Acta 48, 615-621 (2003).

36. Jun Chen, Yi Huang, Nannan Zhang, Haiyang Zou, Ruiyuan Liu, Changyuan Tao, Xing Fan \& Zhong Lin Wang, Micro-cable structured textile for simultaneously harvesting solar and mechanical energy, Nat. Energy 1,2016, 16138.

37. Guangmin Zhou, Feng Lia and Hui-Ming Cheng, Progress in flexible lithium batteries and future prospects, Energy Environ. Sci. 2014, 7, 1307.

38. Guangmin Zhou, Jie Sun, Yang Jin, Wei Chen, Chenxi Zu, Rufan Zhang, Yongcai Qiu, Jie Zhao, Denys Zhuo, Yayuan Liu, Xinyong Tao, Wei Liu, Kai Yan, Hye Ryoung Lee, Yi CuiSulfiphilic Nickel Phosphosulfide Enabled Li2S Impregnation in 3D Graphene Cages for Li-S Batteries, Adv. Mater. 2017, 29, 1603366.

39. Nannan Zhang, Jun Chen, Yi Huang, Wanwan Guo, Jin Yang, Jun Du, Xing Fan, Changyuan Tao, A Wearable All-Solid Photovoltaic Textile, Adv. Mater. 28, 2016, 263. 